\title{
Incidence, prevalence, mortality, and causes of death in Waldenström macroglobulinemia: a nationwide, population-based cohort study
}

\author{
Seri Jeong ${ }^{1}$, Seom Gim Kong ${ }^{2}$, Da Jung Kim³ ${ }^{3}$ Sangjin Lee ${ }^{4}$ and Ho Sup Lee ${ }^{3^{*}}$ (D)
}

\begin{abstract}
Background: The epidemiological features of Waldenström macroglobulinemia (WM) have seldom been investigated at a national level, particularly in East Asia. The goal of our study is to present the incidence, prevalence, mortality, survival with competing risks, and causes of death of patients with WM.

Methods: We used a national population-based database, operated by the Health Insurance Review and Assessment Service of the Korean government. This data includes information on all WM patients diagnosed according to uniform criteria, between 2003 and 2016.

Results: The total number of patients newly diagnosed with WM during the study period was 427, with a male-tofemale ratio of 3.2:1. The incidence increased from 0.03 to 0.10 per $10^{5}$ between 2003 and 2016, and the prevalence was 0.42 per $10^{5}$ in 2016. A total of 217 patients with WM died during the study period (standardized mortality ratio $=7.57$ ), and the overall survival (OS) of WM patients was $47.5 \%$. On multivariate analysis, older age was associated with worse OS ( $P<0.0001)$. WM was the most common cause of death $(n=102,48.6 \%)$, followed by other malignant neoplasms ( $n=82,39.0 \%)$.

Conclusions: The national incidence of WM in Korea, a racially homogeneous country in Asia, was lower than that in previous reports from other countries, reflecting ethnic disparities. However, the incidence increased, and mortality was the highest ever reported. The main cause of death was WM in itself. This study reflects the need for greater awareness of WM, particularly in Asian countries.
\end{abstract}

Keywords: Waldenström macroglobulinemia, Epidemiology, Incidence, Mortality, Survival

\section{Background}

Waldenström macroglobulinemia (WM) is an indolent B-cell malignancy characterized by the presence of immunoglobulin $\mathrm{M}$ (IgM) monoclonal gammopathy and lymphoplasmacytic bone marrow infiltration [1].

\footnotetext{
* Correspondence: hs52silver@gmail.com

${ }^{3}$ Division of Hematology-Oncology, Department of Internal Medicine, Kosin University College of Medicine, 262, Gamcheon-ro, Seo-gu, Busan 49267, South Korea

Full list of author information is available at the end of the article
}

WM represents nearly $2 \%$ of all hematologic malignancies with 1000 to 1500 newly diagnosed cases per year in the United States based on the Surveillance, Epidemiology and End Results (SEER) database $[2,3]$.

Owing to the diverse clinical course of WM, treatment is not necessary for some patients at diagnosis. However, most patients with WM require systemic treatment at some time during the course of their disease [4]. The advent of new treatments, such as

(c) The Author(s). 2020 Open Access This article is licensed under a Creative Commons Attribution 4.0 International License, which permits use, sharing, adaptation, distribution and reproduction in any medium or format, as long as you give appropriate credit to the original author(s) and the source, provide a link to the Creative Commons licence, and indicate if changes were made. The images or other third party material in this article are included in the article's Creative Commons licence, unless indicated otherwise in a credit line to the material. If material is not included in the article's Creative Commons licence and your intended use is not permitted by statutory regulation or exceeds the permitted use, you will need to obtain permission directly from the copyright holder. To view a copy of this licence, visit http://creativecommons.org/licenses/by/4.0/ The Creative Commons Public Domain Dedication waiver (http://creativecommons.org/publicdomain/zero/1.0/) applies to the data made available in this article, unless otherwise stated in a credit line to the data. 
monoclonal antibodies, proteasome inhibitors, and Bruton tyrosine kinase inhibitors, with better understanding of the pathogenesis, have improved the expected clinical outcomes of WM $[5,6]$. However, WM still remains incurable, and its management is becoming increasingly complex $[3,4]$.

According to population-based studies, the survival trends in patients with WM have improved within the last decade [7, 8]. However, variations in geographical location and ethnicity affect the survival of WM patients [8-10]. Other than in Europe and the United States, the epidemiological characteristics of WM have rarely been reported. In addition, only small numbers of Asian patients had been included in previously reported studies.

The present study was based on a comprehensive database, which is run by the National Health Insurance (NHI) of the Korean government. This database contains all the records of healthcare utilization among inpatients and outpatients. Therefore, this database was considered appropriate to reliably investigate the epidemiological features of WM.

Using this database, we performed a comprehensive population-based analysis to investigate the incidence, prevalence, mortality, survival with competing risks, and the causes of death in WM patients in the entire Korean population.

\section{Methods}

\section{Data source}

The data used in this study was extracted from the Health Insurance Review and Assessment Service (HIRA) database, which is based on data from a NHI system run by the Korean government. Healthcare institutions submit the medical data of all inpatient and outpatients in electronic format to the HIRA for reimbursement purposes. The claims data integrated by HIRA include all healthcare utilization information on inpatients and outpatients. Information regarding patient demographics, principal diagnosis, comorbidities, prescription history, and performed procedures is included in this database. The diagnostic information is based on the International Classification of Diseases (ICD), 10th revision. In this study, we obtained all WM cases from the HIRA database registered between January 2003 and the end of December 2016.

The causes of death for the deceased WM patients were also analyzed by linking the Statistics Korea data with the HIRA database. In the Statistics Korea data, the causes of death are documented according to the ICD10 , which are verified by physicians at the time of death and include information regarding all casualties. The number of person-years was calculated from the time of registration to death.

\section{Study population (patient selection)}

All patients who had made claims to HIRA for WM from January 2003 to December 2016 were included in this study. The patients, who were diagnosed with WM based on criteria established by the NHI, were registered in the HIRA database. The criteria are similar to that of the criteria for WM described by Owen et al. [1]. The criteria for WM, defined by the ICD-10 code C88.0, are as follows: IgM monoclonal gammopathy, bone marrow infiltration by small lymphocytes, plasmacytoid cells and plasma cells, and typical immunophenotype (positive for surface immunoglobulin, CD19, CD20, and negative for CD5, CD10, and CD23). We excluded patients diagnosed before $2002(n=5)$ to minimize classification bias. The patients with non-IgM lymphoplasmacytic lymphoma and those with incomplete data $(n=33)$ were also excluded. Cases diagnosed only by death certificates were also excluded from the study. Since our study population depends on the ICD-10 code determined by physicians, it was difficult to completely rule out rare conditions such as IgM secreting marginal zone lymphoma and IgM plasma cell myeloma from WM.

The records of medical visits, demographic information, and death status were collected from the HIRA database for all patients diagnosed with WM. Patients included in this study were followed until the end of December 2016 to determine their vital status and causes of their deaths.

\section{Statistical analysis}

We evaluated the incidence, prevalence, mortality, and survival rates of WM patients in Korea from 2003 to 2016. An incident case was defined as a patient who had been newly diagnosed and registered as having WM in the HIRA database during a corresponding year. A oneyear washout period was applied to prevent prevalent cases from interfering with our data. Any patient free of any WM-related claims for at least one year in the HIRA database was defined as a new WM case in the washout period. The first date claimed in the HIRA database as a WM patient, was designated as the date of initial diagnosis. We calculated the incidence rate by dividing the number of total incident WM patients by the entire Korean population in the middle (mostly July) of each corresponding year.

A prevalent case was defined as a person who was registered in the HIRA database as a WM patient. Prevalent cases included all patients who had been diagnosed with WM during the corresponding year. Patients, who were registered as incident cases during previous years, were also included. The prevalence rates were calculated by dividing the number of total prevalent WM cases by the entire Korean population in each corresponding year. When calculating 
prevalence rates, those who died in the previous years were excluded from prevalent cases. The average ageand gender-specific incidence and prevalence rates were also calculated by dividing the number of WM cases in each specific age and gender group by the age- and gender-specific entire Korean population and averaging these data during the study period.

We calculated the annual mortality by dividing the number of total WM patients who died during that year by the entire number of WM patients in the HIRA database for the corresponding year. The mortality was compared with that of the general population listed in Statistics Korea using the SMR (standardized mortality ratio) with 95\% CIs (confidence intervals). The SMR is defined as the ratio of patients who died from WM relative to the entire number of deaths in the general population. We calculated the SMR by dividing observed deaths by expected deaths derived from the mortality of the entire Korean population archived in Statistics Korea.

Survival information from the HIRA database was used in survival analysis. The Kaplan-Meier method with log-rank test was used to estimate the survival curves. The date of initial registration was regarded as the date of initial diagnosis. The person-years for WM patients were accumulated at the time of entry in the study until death. We also performed a subgroup analysis of survival by epoch of diagnosis, age, and treatment groups. Multivariate Cox proportionalhazards regression models were also used to examine the variables related to survival rates. The causes of death were analyzed for all WM mortality cases and presented according to the major disease classifications.

Statistical analyses were performed using $\mathrm{R}$ statistical software (version 3.4.4, R Foundation for Statistical Computing, Vienna, Austria) and SAS statistical analysis software (version 9.4, SAS Institute Inc., Cary, NC, USA). $P$ values less than 0.05 were considered statistically significant.

\section{Results}

\section{Incidence of WM}

The overall incidence and the age- and gender-specific annual incidence of WM are presented in Table 1 and Fig. 1a. The total number of patients with WM in Korea between 2003 and 2016 was 427, comprising 326 men and 101 women. The number of incident cases was 14 to 53 , and the annual incidence increased from 0.03 to 0.10 per 100,000 people from 2003 through 2016. The median patient-age in these cases was 70.0 years (1st to 3rd quartile range: 61.0 to 76.0 years), and the median ages for men and women was 70.0 (1st to 3rd quartile range: 62.0 to 76.0 years), and 69.0 years (1st to 3 rd quartile range: 58.0 to 76.0 years), respectively. The incidence rates by gender were 0.09 per 100,000 individuals for men and 0.03 per 100,000 individuals for women, rendering a male-to-female ratio of 3.2:1.

Age-specific incidence is shown in Fig. 1a. Among all age groups, the highest incidence rate of WM was observed in people aged between 75 and 79 years $(8.15$ per 100,000 individuals), accounting for $19.7 \%$ of all incident cases. The

Table 1 Incidence and prevalence of Waldenström macroglobulinemia between 2003 and 2016

\begin{tabular}{|c|c|c|c|c|c|c|c|c|c|c|c|c|}
\hline \multirow[t]{2}{*}{ Year } & \multicolumn{3}{|c|}{ Incident cases } & \multicolumn{3}{|c|}{ Prevalent cases } & \multicolumn{3}{|c|}{ Incidence per $10^{5} / y^{a}$} & \multicolumn{3}{|c|}{ Prevalence per $10^{5} / \mathrm{y}^{\mathrm{b}}$} \\
\hline & Total & Male & Female & Total & Male & Female & Total & Male & Female & Total & Male & Female \\
\hline 2003 & 14 & 8 & 6 & 19 & 12 & 7 & 0.03 & 0.03 & 0.02 & 0.04 & 0.05 & 0.03 \\
\hline 2004 & 18 & 16 & 2 & 33 & 26 & 7 & 0.04 & 0.07 & 0.01 & 0.07 & 0.11 & 0.03 \\
\hline 2005 & 15 & 13 & 2 & 45 & 36 & 9 & 0.03 & 0.05 & 0.01 & 0.09 & 0.15 & 0.04 \\
\hline 2006 & 19 & 15 & 4 & 57 & 45 & 12 & 0.04 & 0.06 & 0.02 & 0.12 & 0.18 & 0.05 \\
\hline 2007 & 21 & 20 & 1 & 66 & 55 & 11 & 0.04 & 0.08 & 0.00 & 0.13 & 0.22 & 0.04 \\
\hline 2008 & 25 & 17 & 8 & 81 & 63 & 18 & 0.05 & 0.07 & 0.03 & 0.16 & 0.25 & 0.07 \\
\hline 2009 & 28 & 22 & 6 & 94 & 71 & 23 & 0.06 & 0.09 & 0.02 & 0.19 & 0.28 & 0.09 \\
\hline 2010 & 25 & 17 & 8 & 107 & 77 & 30 & 0.05 & 0.07 & 0.03 & 0.21 & 0.30 & 0.12 \\
\hline 2011 & 39 & 28 & 11 & 132 & 99 & 33 & 0.08 & 0.11 & 0.04 & 0.26 & 0.39 & 0.13 \\
\hline 2012 & 45 & 37 & 8 & 149 & 115 & 34 & 0.09 & 0.15 & 0.03 & 0.29 & 0.45 & 0.13 \\
\hline 2013 & 37 & 27 & 10 & 162 & 122 & 40 & 0.07 & 0.11 & 0.04 & 0.32 & 0.48 & 0.16 \\
\hline 2014 & 43 & 29 & 14 & 182 & 136 & 46 & 0.08 & 0.11 & 0.05 & 0.35 & 0.53 & 0.18 \\
\hline 2015 & 45 & 36 & 9 & 202 & 151 & 51 & 0.09 & 0.14 & 0.03 & 0.39 & 0.59 & 0.20 \\
\hline 2016 & 53 & 41 & 12 & 216 & 161 & 55 & 0.10 & 0.16 & 0.05 & 0.42 & 0.62 & 0.21 \\
\hline Total & 427 & 326 & 101 & 1545 & 1169 & 376 & 0.06 & 0.09 & 0.03 & 0.22 & 0.33 & 0.11 \\
\hline
\end{tabular}

ancidence $=$ (incident cases/total Korean population) $\times 10^{5}$

${ }^{\mathrm{b}}$ Prevalence $=($ prevalent cases $/$ total Korean population $) \times 10^{5}$ 


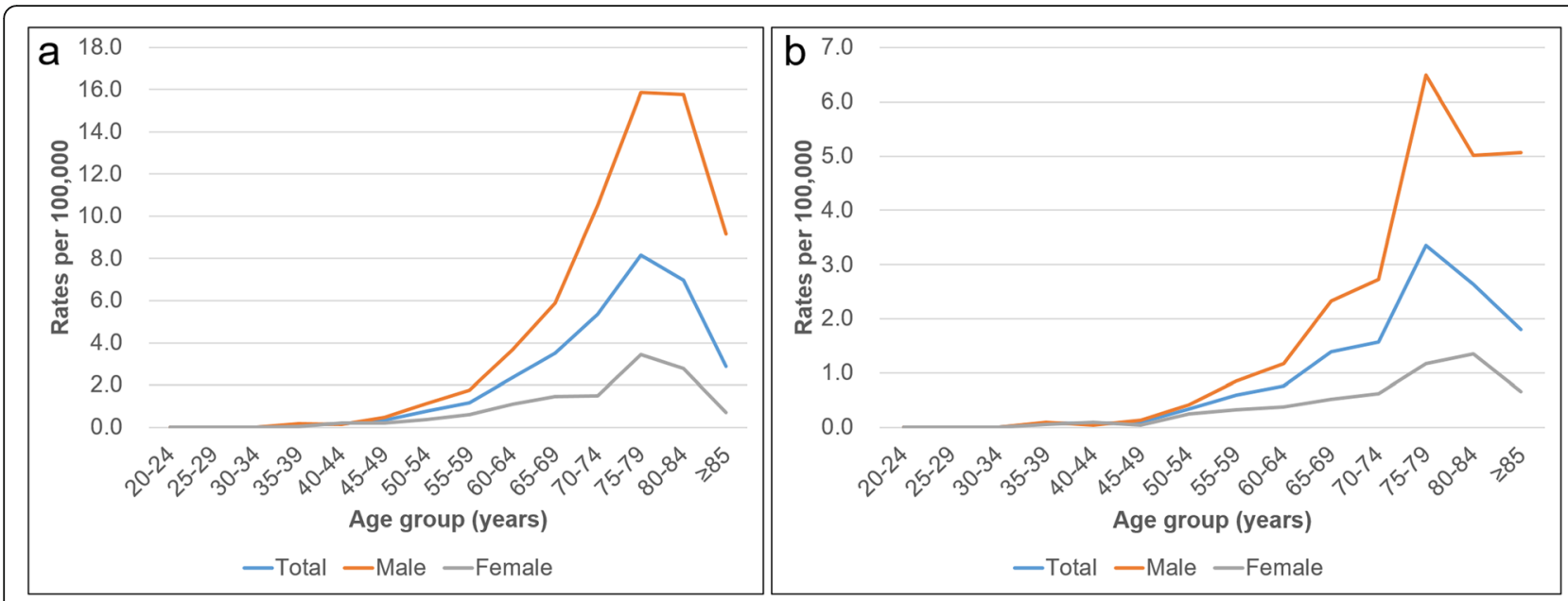

Fig. 1 Incidence and prevalence of Waldenström macroglobulinemia by age and gender. Vertical axis shows incidence rates in $10^{5}$ people; horizontal axis presents age in 5-year increments until the age of 85 years. a Incidence $\mathbf{b}$ prevalence

highest incidence peak for men (from 75 to 79 years old) was similar to that for women (Additional file 1: Table S1).

\section{Prevalence of WM}

The annual prevalence and age- and gender-specific prevalence of WM are shown in Table 1 and Fig. $1 \mathrm{~b}$. The crude prevalence was 0.42 per 100,000 individuals, when calculated with the 2016 population as the denominator. The prevalence for men and women in 2016 were 0.62 and 0.21 , respectively; the male-tofemale ratio was 2.9:1, which was similar to that of incidence (Additional file 2: Table S2). On the other hand, the prevalence in 2003 was 0.04 per 100,000 individuals, with an 11.4-fold increase during the study period (13.4-fold for men and 7.9-fold for women). The annual increase rate between 2003 and 2016 was $18.96 \%$ (20.38\% for men and $15.86 \%$ for women).

As shown in Fig. 1b, the shape of the prevalence plot was comparable to that of the incidence plot. The prevalence peak occurred at ages 75 to 79, accounting for $22.7 \%$ of all prevalent cases (Additional file 2: Table S2). The similar age group also exhibited the highest prevalence for both males and females.

\section{Mortality}

In total, 217 of the 427 incident WM patients comprising 169 males and 48 females, died during the study period. The overall average annual mortality was 126.68 per 1000 individuals; 131.42 per 1000 males, and 116.38 per 1000 females. The age- and gender-specific SMRs for WM are shown in Table 2. The SMR for all WM patients was 7.57 (95\% CI, 6.56 to 8.57 ), indicating that mortality in WM patients was significantly higher compared to that of the general population. The SMR for females $(9.99 ; 95 \%$ CI, 7.16 to 12.82$)$ was higher than that of males (5.56; $95 \% \mathrm{CI}, 4.72$ to 6.39 ).

\section{Survival}

The survival curve for WM patients is illustrated in Fig. 2. We followed 427 incident cases of WM from the time of diagnosis until December 31, 2016, which is equivalent to a total of 1368 person-years. In total, 217 incident cases died after a median follow-up of 4.5 years. The 14-year overall survival (OS) rate of WM patients was $47.5 \%$ (46.6\% for males and $50.5 \%$ for females). The median OS of them was 4.5 years $(95 \% \mathrm{CI}, 3.6$ to 5.5 years). The 2-, 5-, and 10-year survival rates were 69.0,

Table 2 Age- and gender- specific standard mortality ratios and mortality of Waldenström macroglobulinemia

\begin{tabular}{|c|c|c|c|c|c|c|c|c|c|}
\hline \multirow{2}{*}{$\begin{array}{l}\text { Age } \\
\text { (years) }\end{array}$} & \multicolumn{2}{|c|}{ Total } & \multirow[b]{2}{*}{ SMR $(95 \% \mathrm{Cl})$} & \multicolumn{2}{|c|}{ Male } & \multirow[b]{2}{*}{ SMR (95\% Cl) } & \multicolumn{2}{|c|}{ Female } & \multirow[b]{2}{*}{ SMR $(95 \% \mathrm{CI})$} \\
\hline & $\mathrm{O}$ & E & & $\mathrm{O}$ & E & & $\mathrm{O}$ & E & \\
\hline $20-49$ & 1 & 0.18 & $5.46(0.27-26.93)$ & 1 & 0.15 & $6.47(0.32-31.91)$ & 0 & 0.04 & $0.00(-)$ \\
\hline $50-59$ & 8 & 1.14 & $7.03(3.27-13.35)$ & 5 & 1.21 & $4.12(1.51-9.13)$ & 3 & 0.16 & $18.32(4.66-49.87)$ \\
\hline $60-69$ & 39 & 4.34 & $8.99(6.48-12.17)$ & 29 & 5.10 & 5.69 (3.88-8.06) & 10 & 0.50 & $20.02(10.17-35.68)$ \\
\hline 70-79 & 75 & 13.92 & $5.39(4.27-6.72)$ & 60 & 15.85 & $3.78(2.91-4.84)$ & 15 & 1.88 & $7.97(4.63-12.85)$ \\
\hline$>80$ & 94 & 9.11 & $10.32(8.39-12.57)$ & 74 & 8.09 & $9.14(7.23-11.41)$ & 20 & 2.22 & $9.03(5.67-13.69)$ \\
\hline Total & 217 & 28.68 & $7.57(6.56-8.57)$ & 169 & 30.41 & $5.56(4.72-6.39)$ & 48 & 4.80 & $9.99(7.16-12.82)$ \\
\hline
\end{tabular}

$O$ number of observed deaths, $E$ number of expected deaths, SMR standard mortality ratio, $C l$ confidence interval 

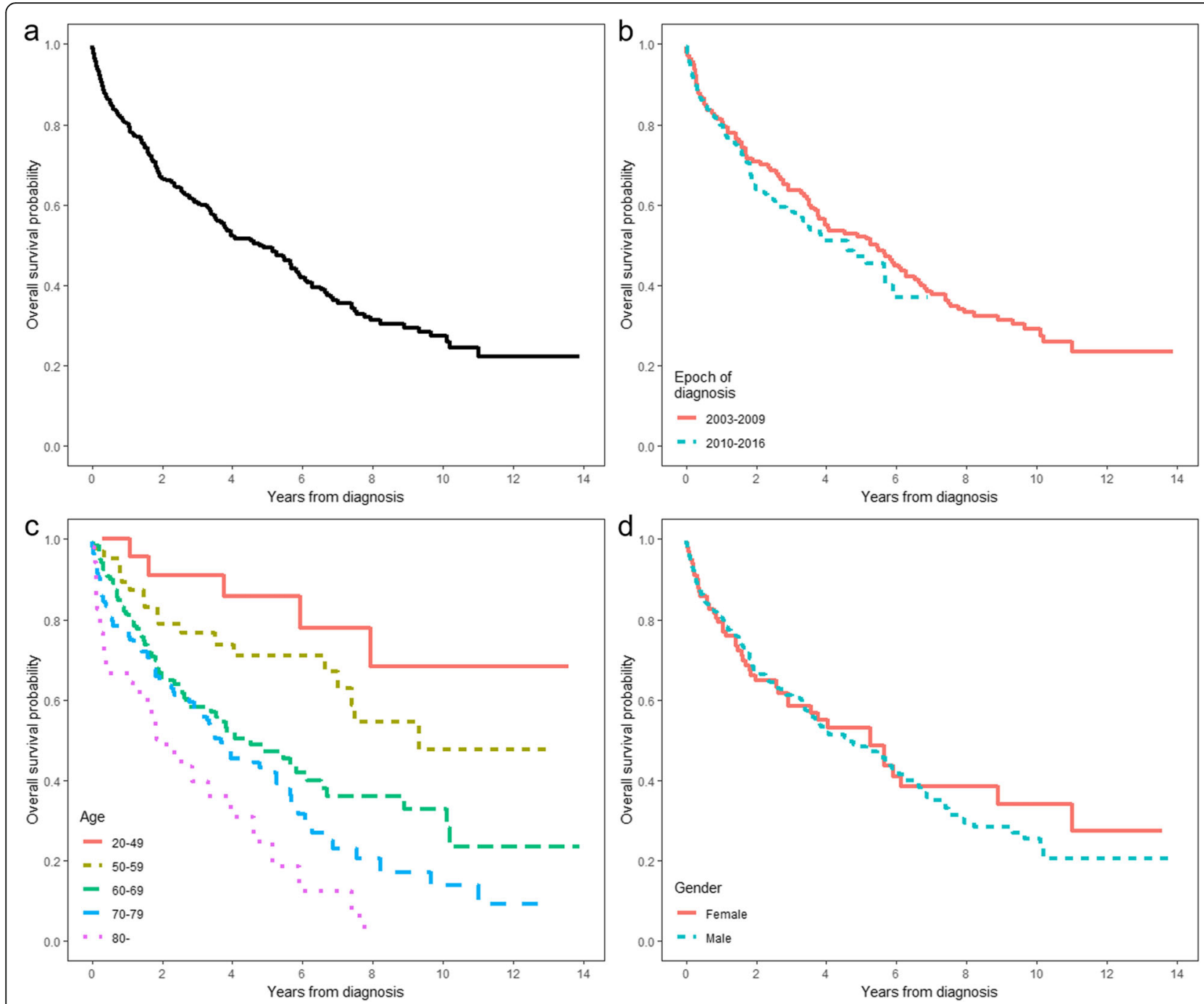

Fig. 2 Overall survival in patients with Waldenström macroglobulinemia. a entire cohort $\mathbf{b}$ epoch of diagnosis $\mathbf{c}$ age categories $\mathbf{d}$ gender

Table 3 Multivariate analysis for overall survival in patients with Waldenström macroglobulinemia

\begin{tabular}{|c|c|c|c|c|c|c|c|c|c|c|c|c|c|}
\hline Variable & Number (\%) & 2-year HR & $95 \% \mathrm{Cl}$ & $P$ & 5-year HR & $95 \% \mathrm{Cl}$ & P & 10-year HR & $95 \% \mathrm{Cl}$ & $P$ & Total HR & $95 \% \mathrm{Cl}$ & $P$ \\
\hline \multicolumn{14}{|l|}{ Age (years) } \\
\hline $20-49$ & $26(6.09)$ & Reference & & & Reference & & & Reference & & & & Reference & \\
\hline $50-59$ & $63(14.75)$ & 2.61 & $0.58-11.78$ & 0.21 & 2.43 & $0.70-8.48$ & 0.16 & 2.22 & $0.83-5.89$ & 0.11 & 2.30 & $0.86-6.12$ & 0.10 \\
\hline $60-69$ & $121(28.34)$ & 4.99 & $1.20-20.67$ & 0.03 & 5.28 & $1.65-16.93$ & 0.01 & 4.06 & $1.62-10.13$ & 0.00 & 4.34 & $1.74-10.84$ & $<0.01$ \\
\hline $70-79$ & 165 (38.64) & 5.32 & $1.29-21.86$ & 0.02 & 5.90 & $1.86-18.74$ & 0.00 & 5.49 & $2.22-13.56$ & 0.00 & 5.82 & $2.36-14.39$ & $<0.01$ \\
\hline$>80$ & $52(12.18)$ & 8.21 & $1.92-34.99$ & 0.00 & 9.46 & $2.87-31.14$ & 0.00 & 8.98 & $3.48-23.17$ & 0.00 & 9.57 & $3.70-24.72$ & $<0.01$ \\
\hline \multicolumn{14}{|l|}{ Gender } \\
\hline Female & $101(23.65)$ & Reference & & Refer & ence & & & Reference & & & Reference & & \\
\hline Male & $326(76.35)$ & 0.89 & $0.60-1.32$ & 0.57 & 0.98 & $0.69-1.40$ & 0.93 & 0.99 & $0.72-1.36$ & 0.94 & 0.99 & $0.72-1.36$ & 0.94 \\
\hline \multicolumn{14}{|c|}{ Epoch of diagnosis } \\
\hline 2003-2009 & 140 (32.79) & Reference & & Refer & ence & & & Reference & & & Reference & & \\
\hline 2010-2016 & $287(67.21)$ & 1.15 & $0.79-1.68$ & 0.47 & 1.10 & $0.80-1.50$ & 0.56 & 1.15 & $0.85-1.55$ & 0.36 & 1.15 & $0.85-1.56$ & 0.35 \\
\hline
\end{tabular}


57.1, and 48.3\%, respectively (Fig. 2a). For the 2003 to 2009 cohort, the median OS was 5.4 years (95\% CI, 3.7 to 6.5 years). Meanwhile, the median OS was estimated 3.9 years ( $95 \%$ CI, 3.0 to 5.2 years) for the 2010 to 2016 cohort (Fig. 2b). There were statistical differences in the OS based on age $(P<0.01)$. The median OS for patients aged between 50 and 59, 60 and 69, 70 and 79, and $\geq 80$ years were 9.3 years, 4.1 years, 3.5 years, and 1.8 years, respectively (Fig. 2c). The median OS according to the gender was 5.19 years for female, and 4.3 years for male (Fig. 2d). The specific chemotherapy agents administered to Korean patients are shown in Additional file 3: Table S3.

\section{Multivariate analysis for competing risks}

On multivariate analysis for OS (Table 3), the factor significantly related to worse OS was older age $(P<0.01)$. The total hazard ratios (HR) for WM patients aged between 50 and 59, 60 and 69, 70 and 79, and $\geq 80$ years, compared with patients between 20 and 49 years, were $2.30 \quad(P=0.10), 4.34 \quad(P<0.01)$, $5.82(P<0.01)$, and $9.57(P<0.01)$, respectively. In contrast, the gender $(P=0.94)$, and epoch of diagnosis $(P=0.35)$ were not significantly associated with OS.

\section{Causes of death}

The causes of deaths of WM patients are summarized in Table 4. WM-related deaths were recorded as the most common cause, accounting for 102 cases (48.57\%). Malignant neoplasms constituted the second most common cause of death, being responsible for 82 deaths (39.05\%). Among the 82 deaths due to malignant neoplasms, 23 were due to non-follicular lymphoma, and 22 were due to malignant plasma cell neoplasms.

\section{Discussion}

WM is an uncommon B-cell non-Hodgkin lymphoma, on which few epidemiological studies have been performed. In this study, the average 14-year incidence was 0.06 per 100,000 people. The incidence was stable between 1988 and 2005, at 0.3 per 100,000 people in the United States, and 0.55 per 100,000 people between 1999 and 2001 in England, which were significantly higher than the values found in this study $[2,11]$. Differences in ethnicity, and geographic variations, exert a considerable influence on the lower incidence of WM in Korea. According to a previous report based on the SEER database, the incidence was higher in whites (0.41 per 100,000 people) than in nonwhites ( 0.20 per 100,000 people), suggesting distinct geographic differences [12]. Additionally, similar to the results of our study, the incidence of WM in Japan (0.043 per 100,000 person-years) and Taiwan (0.031 per 100,000 person-years) between 1996 and 2003, were lower than those of Asian ethnic groups (0.10 to 0.32 ) based on SEER data $[13,14]$. These reports provide evidence that racial differences exist in the incidence of WM, and that environmental and/or genetic factors may have an influence on the development of WM. These results are also supported by the fact that the prevalence of IgM-monoclonal gammopathy of undetermined significance (MGUS), which is a precursor state to WM, is lower in the Asian

Table 4 Cause of death among patients with Waldenström macroglobulinemia in Korea

\begin{tabular}{|c|c|c|c|c|c|}
\hline Condition (ICD-10 code) & Male & Female & 2003-2009 & 2010-2016 & Total $^{a}(\%)$ \\
\hline \multicolumn{6}{|l|}{ Neoplasm } \\
\hline Malignant neoplasm (C00-C97 except for C88) & 63 & 19 & 33 & 49 & $82(39.05)$ \\
\hline Waldenström macroglobulinemia (C88) & 83 & 19 & 54 & 48 & $102(48.57)$ \\
\hline Other neoplasm (D00-D48) & 1 & 0 & 0 & 1 & $1(0.48)$ \\
\hline Hematology (D50-D89) & 2 & 1 & 1 & 2 & $3(1.43)$ \\
\hline \multicolumn{6}{|l|}{ Circulatory system } \\
\hline Cardiovascular (100-152) & 2 & 2 & 1 & 3 & $4(1.90)$ \\
\hline Cerebrovascular (160-169) & 1 & 1 & 0 & 2 & $2(0.95)$ \\
\hline Infection (A00-B99) & 0 & 2 & 1 & 1 & $2(0.95)$ \\
\hline Respiratory system (J00-J99) & 4 & 1 & 2 & 3 & $5(2.38)$ \\
\hline Digestive system (K00-K93) & 1 & 0 & 0 & 1 & $1(0.48)$ \\
\hline Musculoskeletal system and connective tissue (M00-M99) & 1 & 0 & 1 & 0 & $1(0.48)$ \\
\hline Trauma (S00-S99, T00-T98, V01-V99, W00-W99, X00-X99, and Y00-Y98) & 2 & 0 & 1 & 1 & $2(0.95)$ \\
\hline Other (R00-R99, and Z00-Z99) & 5 & 0 & 2 & 3 & $5(2.38)$ \\
\hline Total & 165 & 45 & 96 & 114 & $210(100.00)$ \\
\hline
\end{tabular}

${ }^{\mathrm{a} A}$ total of 7 out of 217 patients who died from Waldenström macroglobulinemia had unknown or unspecified causes 
population than in the other races $[15,16]$. Furthermore, genetic predisposition, and immunologic factors in the pathogenesis of WM might be the reason behind these low incidence rates [17-21]. Although Korea may indeed have lower incidence, it should be noted that direct comparison with these studies may not be feasible. The incidence may be affected by the data collection methods and with the lapse of time. Additionally, the diagnostic criteria applied for WM may have been influenced by the cases diagnosed in the previous epoch [2, 4]. The period of our study covered the period during which the consensus panel recommendations for the diagnostic criteria of WM, published in 2003, were applied [1].

The annual incidence of WM has increased from 0.03 to 0.10 per 100,000 people in our study, compared with the consistent incidence reported by previous studies [2, 11, 22]. There are some studies, which showed that geographic alterations and racial differences are associated with significant increase in annual percentage change [12, 23]. This increase would partly be because of the increasing availability of diagnostic tools, and established criteria, which allowed the diagnosis of WM cases that would not have been possible to diagnose in the previous epoch. In addition, the differences between countries, in terms of the sample size, the growth rate of the aging population, and exposure to environmental factors, might explain these phenomena $[12,23]$. Based on the incidence data, a comparatively lower prevalence rate than that of other countries was expected. However, the comparison was impossible because, to the best of our knowledge, no reports for the prevalence of WM were available.

Male dominance has been continuously reported in WM. In our study, the male-to-female ratio was 3.2:1, which is slightly higher than that reported in other studies from the Europe and United States (1.7 to 2.4: 1) $[11,12,14,24,25]$. This study revealed the vulnerability of males in Korea to WM. The male-to-female ratios for WM in Japan (3.1:1) [23] and in Olmsted County (3.1:1) [22] were similar to our value, suggesting regional differences. In regard to the age distribution, the incidence peak occurred in people aged between 75 and 79 years, which is consistent with the values reported for other countries [12, 25].

The SMR of WM patients in our study (7.57) was the highest ever reported. A recent study, conducted in Olmsted County, presented a SMR of 2.4 for the patients diagnosed with WM after 2000 [22]. Another study reported that the SMR of asymptomatic WM was equivalent to that of the general population, while that of symptomatic patients was 5.4 [26].
In respect of survival, the median OS of Korean WM patients ( 4.5 years; $95 \%$ CI, 3.6 to 5.5 years) was significantly lower than that of Asians living in United States (7.4 years for patients diagnosed with WM between 2003 and 2009; 95\% CI, 6.8 to 8.2 years), implicating an environmental effect [9]. Considerably longer median OS (7 to 25 years) has been reported from the Europe and United States owing to ethnic disparities, epoch of diagnosis, and different study designs, such as hospital-based cohort and populationbased research $[4,9,10,27,28]$. The OS of our study $(47.5 \%)$ was also lower than the range reported in previous studies (between 57 and 62\% in England and the United States) $[2,4,11]$. The OS in our study was substantially worse than those of other countries, taking into account the epoch of diagnosis, because the study periods of the others were mostly before 2010.

Although there is no standard of care established for WM, the advent of anti-CD20 monoclonal antibodies, nucleoside analogs, alkylating agents, and proteasome inhibitors have shown high response rates and have resulted in better OS $[3,29,30]$. In particular, rituximab singly or in combination has been commonly used as first-line therapy, and has contributed to better OS (62 to $97.1 \%$ ) in the Western countries [31-34]. However, the HIRA does not reimburse the rituximab-based chemotherapy because of the paucity of studied data for WM patients in Korea. Exclusion from the HIRA system could therefore be the cause for the higher SMR and the poorer survival of Korean patients with WM. It would be necessary to add at least rituximab-based treatment to the reimbursement system of HIRA for improving the outcome of WM patients.

Additionally, we investigated the trends of the mortality rates in the general population to find out the reason why the OS did not improve according to the epoch of diagnosis. However, there were no notable differences of general population which could influence on the mortality of WM patients. The restrictions in the appropriate prescription of rituximab combined chemotherapy owing to reimbursement issues could have contributed to the lack of improvement. Furthermore, a relatively short follow-up period, considering the expected longer life span of WM patients [27], in addition to an inevitable small sample size compared to other populationbased studies [4, 7], may have affected these survival results.

Our competing risk analysis showed that age was a strong factor $(P<0.01)$ for OS in patients with WM. The significant association between old age and poorer outcome was persistently reported in previous studies $[2,4,11]$. 
The main cause of death in our cohort was directly attributable to WM, and the magnitude (48.57\%) was almost two times higher than that of a previous report from the SEER database (25\%) [4]. The poorer survival of Korean patients owing to obstacles in the reimbursement system to newly introduced therapies could be associated with higher WM-related deaths. The next most common cause of death was non-follicular lymphoma, which was consistent with the higher proportion of deaths due to lymphoma in the SEER database [4]. The third most common cause was malignant plasma cell neoplasms, implicating considerable relevance to WM [35]. Because our data based on the records archived in Statistics Korea, it was difficult to differentiate nonfollicular lymphoma, and plasma cell neoplasm from WM. The possibility of misclassification should be considered.

This study had several limitations. The lack of detailed clinical information such as the symptoms, the laboratory data, or genetic features, led to restrictions on the adjustments of severity for each WM patient. Moreover, classification bias could exist because we used registry data based on physicians' diagnoses without additional pathological confirmation. Despite these limitations, the strength of our study is the use of a nationwide population database of recent WM patients, after application of established diagnostic criteria. To the best of our knowledge, no other study has reported on the incidence, prevalence, mortality, and causes of death using a recent and nationwide data source, especially in Asia. The relatively large sample size covering the entire national population and unbiased measures used in this study could provide reliable information on WM patients.

\section{Conclusions}

In conclusion, we are the first to report the epidemiologic features of WM based on the recent nationwide database of Korea, a racially homogeneous country of East Asia. This study confirmed the low incidence of WM in Korea, reflecting ethnic disparities. However, the incidence increased three fold between 2003 and 2016, and mortality was the highest ever reported. The most common cause of death was directly attributable to WM. This study indicates that more attention needs to be paid to WM patients, particularly in Eastern Asian countries.

\section{Supplementary information}

Supplementary information accompanies this paper at https://doi.org/10. 1186/s12885-020-07120-9.

Additional file 1: Table S1. Age-specific annual incidence of Waldenström macroglobulinemia between 2003 and 2016.
Additional file 2: Table S2. Age-specific prevalence of Waldenström macroglobulinemia in Korea, 2016.

Additional file 3: Table S3. The specific chemotherapy agents administered to Korean patients with Waldenström macroglobulinemia.

\section{Abbreviations}

Cl: Confidence interval; HIRA: Health Insurance Review and Assessment Service; HR: Hazard ratio; ICD: International Classification of Diseases; IgM: Immunoglobulin M; MGUS: IgM-monoclonal gammopathy of undetermined significance; OS: Overall survival; SEER: Surveillance, Epidemiology and End Results; SMR: Standardized mortality ratio; WM: Waldenström macroglobulinemia

\section{Acknowledgements}

The authors acknowledge the efforts of the staff of the HIRA database, which is supported by the NHI system of Korea, for the maintenance and extraction of precise WM data as a research resource. We also thank Hyun Jung Kim and Hyeong Sik Ahn, the staff of the Department of Preventive Medicine, College of Medicine, Korea University, for their assistance in preparing this article.

\section{Authors' contributions}

SJ was a major contributor in conceptualization, design, data collection, analysis and interpretation and wrote the manuscript. SGK was involved in data collection and reviewed the manuscript for hematological content. DJK collected and interpreted the data regarding the hematologic information. SL was a major contributor in collecting, analyzing, and interpretation data regarding statistical information. HSL was a major contributor in designing this study and interpretation data regarding the hematologic information. All authors read and approved the final manuscript.

\section{Funding}

This work was supported by the National Research Foundation of Korea (NRF) grant, funded by the Korean government (Ministry of Science, ICT \& Future Planning)[NRF-2017R1C1B2004597]. The funder had no role in study design, in the collection, analysis and interpretation of data, in the writing of the report, and in the decision to submit the article for publication.

\section{Availability of data and materials}

The datasets limited to anonymisable information and supporting the conclusions of this article are included within this article (Tables, Figures, and Additional files). The repository data for public release is not available because of the personally identifiable information. The full dataset includes clinic centers in which they attend, insurance conditions. Therefore, concerning privacy risks, the data is managed by authorized executive supervisor. If one researcher asks to access data, the researcher should submit the security memorandum and pledge to the Institutional Review Board of National Health Insurance Sharing Service. After approval, the person in charge releases data with blind identification for the discrete requirements and the data should be analyzed only in permitted rooms in centers of National Health Insurance Service. The other researchers could access these data in the same manner as the authors and the authors did not have any special access privileges. Contact information for a data access committee is listed as follows: National Health Insurance Sharing Service, Tel: 82-33-736-2432; Official internet site: https://nhiss.nhis.or.kr/bd/ay/bdaya001 iv.do.

\section{Ethics approval and consent to participate}

This study was approved by the independent Institutional Review Board of Kosin University Gospel Hospital (KUGH 2017-11-027) and conducted in accordance with the Declaration of Helsinki. This study was exempted from the requirement for informed patient consent because personal information was protected and kept anonymous.

Consent for publication

Not applicable.

Competing interests

The authors declare that they have no conflict of interests. 


\section{Author details}

'Department of Laboratory Medicine, Hallym University College of Medicine, 1, Singil-ro, Yeongdeungpo-gu, Seoul 07441 , South Korea. ${ }^{2}$ Department of Pediatrics, Kosin University College of Medicine, 262, Gamcheon-ro, Seo-gu, Busan 49267, South Korea. ${ }^{3}$ Division of Hematology-Oncology, Department of Internal Medicine, Kosin University College of Medicine, 262,

Gamcheon-ro, Seo-gu, Busan 49267, South Korea. ${ }^{4}$ Department of Statistics, Graduate School, Pusan National University, 2, Busandaehak-ro 63 beon-gil, Geumjeong-gu, Busan 46241, South Korea.

Received: 1 April 2019 Accepted: 28 June 2020

Published online: 03 July 2020

\section{References}

1. Owen RG, Treon SP, Al-Katib A, Fonseca R, Greipp PR, McMaster ML, Morra E, Pangalis GA, San Miguel JF, Branagan AR, et al. Clinicopathological definition of Waldenstrom's macroglobulinemia: consensus panel recommendations from the second international workshop on Waldenstrom's Macroglobulinemia. Semin Oncol. 2003;30:110-5..

2. Sekhar J, Sanfilippo K, Zhang Q, Trinkaus K, Vij R, Morgensztern D. Waldenström macroglobulinemia: a surveillance, epidemiology, and end results database review from 1988 to 2005. Leuk Lymphoma. 2012;53:1625-6.

3. Yun S, Johnson AC, Okolo ON, Arnold SJ, McBride A, Zhang L, Baz RC, Anwer F. Waldenstrom Macroglobulinemia: review of pathogenesis and management. Clin Lymphoma Myeloma Leuk. 2017;17:252-62.

4. Castillo JJ, Olszewski AJ, Kanan S, Meid K, Hunter ZR, Treon SP. Overall survival and competing risks of death in patients with Waldenström macroglobulinaemia: an analysis of the surveillance, epidemiology and end results database. Br J Haematol. 2015;169:81-9.

5. Liu YC, Yang YH, Hsiao HH, Yang WC, Liu TC, Chang CS, Yang MY, Lin PM, Hsu JF, Chang PY, et al. Herpes zoster is associated with an increased risk of subsequent lymphoid malignancies - a nationwide population-based matched-control study in Taiwan. BMC Cancer. 2012;12:503.

6. Ma J, Wang S, Zhao M, Deng XS, Lee CK, Yu XD, Liu B. Therapeutic potential of cladribine in combination with STAT3 inhibitor against multiple myeloma. BMC Cancer. 2011;11:255.

7. Kristinsson SY, Eloranta S, Dickman PW, Andersson TM-L, Turesson I, Landgren O, Björkholm M. Patterns of survival in lymphoplasmacytic lymphoma/waldenström macroglobulinemia: a population-based study of 1,555 patients diagnosed in Sweden from 1980 to 2005. Am J Hematol. 2013;88:60-5.

8. Castillo JJ, Olszewski AJ, Cronin AM, Hunter ZR, Treon SP. Survival trends in Waldenstrom macroglobulinemia: an analysis of the surveillance, Epidemiology and End Results database. Blood. 2014;123:3999-4000.

9. Ailawadhi S, Kardosh A, Yang D, Cozen W, Patel G, Alamgir MA, ChananKhan AA. Outcome disparities among ethnic subgroups of Waldenström's Macroglobulinemia: a population-based study. Oncology. 2014;86:253-62.

10. Kastritis E, Kyrtsonis MC, Hatjiharissi E, Symeonidis A, Michalis E, Repoussis P, Tsatalas K, Michael M, Sioni A, Kartasis Z, et al. No significant improvement in the outcome of patients with Waldenström's macroglobulinemia treated over the last 25 years. Am J Hematol. 2011;86:479-83.

11. Phekoo KJ, Jack RH, Davies E, Møller H, Schey SA. The incidence and survival of Waldenström's Macroglobulinaemia in south East England. Leuk Res. 2008;32:55-9.

12. Wang H, Chen Y, Li F, Delasalle K, Wang J, Alexanian R, Kwak L, Rustveld L, Du XL, Wang M. Temporal and geographic variations of Waldenstrom macroglobulinemia incidence: a large population-based study. Cancer. 2012; 118:3793-800

13. Carreon JD, Morton LM, Devesa SS, Clarke CA, Gomez SL, Glaser SL, Sakoda LC, Linet MS, Wang SS. Incidence of lymphoid neoplasms by subtype among six Asian ethnic groups in the United States, 1996-2004. Cancer Causes Control. 2008;19(10):1171-81.

14. Morton LM, Wang SS, Devesa SS, Hartge P, Weisenburger DD, Linet MS. Lymphoma incidence patterns by WHO subtype in the United States, 19922001. Blood. 2006;107:265-76.

15. Iwanaga M, Tagawa M, Tsukasaki K, Kamihira S, Tomonaga M. Prevalence of monoclonal Gammopathy of undetermined significance: study of 52,802 persons in Nagasaki City, Japan. Mayo Clinic Proceedings. 2007;82:1474-9.

16. Kyle RA, Therneau TM, Rajkumar SV, Larson DR, Plevak MF, Offord JR, Dispenzieri A, Katzmann JA, Melton $\amalg$. Prevalence of monoclonal Gammopathy of undetermined significance. N Engl J Med. 2006;354:1362-9.
17. Kristinsson SY, Bjo M, Goldin LR, Mcmaster ML, Turesson I, Landgren O. Risk of lymphoproliferative disorders among first-degree relatives of lymphoma/ Waldenström macroglobulinemia patients: a population-based study in Sweden. Blood. 2008;112:3052-6. https://doi.org/10.1182/blood-2008-06162768.

18. Kristinsson SY, Goldin LR, Turesson I, Björkholm M, Landgren O. Familial aggregation of lymphoplasmacytic lymphoma/Waldenström macroglobulinemia with solid tumors and myeloid malignancies. Acta Haematol. 2012;127:173-7.

19. Kristinsson SY, Koshiol J, Björkholm M, Goldin LR, McMaster ML, Turesson I, Landgren O. Immune-related and inflammatory conditions and risk of lymphoplasmacytic lymphoma or waldenström macroglobulinemia. J Natl Cancer Inst. 2010;102:557-67.

20. McMaster ML, Csako G. Protein electrophoresis, immunoelectrophoresis and immunofixation electrophoresis as predictors for high-risk phenotype in familial Waldenström macroglobulinemia. Int J Cancer. 2008;122:1183-8.

21. Neumann F, Pfreundschuh M, Preuss KD, Schormann C, Zwick C, Murawski $\mathrm{N}$, Kubuschok B. CD4+ T cells in chronic autoantigenic stimulation in MGUS, multiple myeloma and Waldenström's macroglobulinemia. Int J Cancer. 2015;137:1076-84.

22. Kyle RA, Larson DR, McPhail ED, Therneau TM, Dispenzieri A, Kumar S, Kapoor P, Cerhan JR, Rajkumar SV. Fifty-year incidence of Waldenström Macroglobulinemia in Olmsted County, Minnesota, from 1961 through 2010: a population-based study with complete case capture and Hematopathologic review. Mayo Clin Proc. 2018;93:739-46.

23. Iwanaga M, Chiang CJ, Soda M, Lai MS, Yang YW, Miyazaki Y, Matsuo K, Matsuda T, Sobue T. Incidence of lymphoplasmacytic lymphoma/ Waldenström's macroglobulinaemia in Japan and Taiwan population-based cancer registries, 1996-2003. Int J Cancer. 2014;134:174-80.

24. Groves FD, Travis LB, Devesa SS, Ries LA, Fraumeni JF. Waldenström's macroglobulinemia: incidence patterns in the United States, 1988-1994. Cancer. 1998;82:1078-81.

25. Herrinton LJ, Weiss NS. Incidence of Waldenstrom ' s macroglobulinemia incidence of Waldenstrom 's Macroglobulinemia. Blood. 1993;82:3148-50.

26. Morel P, Merlini G. Risk stratification in Waldenström macroglobulinemia. Expert Rev Hematol. 2012;5:187-99.

27. Hensel M, Brust J, Plöger C, Schuster D, Memmer ML, Franz-Werner J, Feustel HP, Karcher A, Fuxius S, Mosthaf FA, et al. Excellent long-term survival of 170 patients with Waldenström's macroglobulinemia treated in private oncology practices and a university hospital. Ann Hematol. 2012;91:1923-8.

28. Kyrtsonis MC, Vassilakopoulos T, Angelopoulou M, Siakantaris M, Kontopidou F, Dimopoulou M, Boussiotis V, Gribabis D, Konstantopoulos K, Vaiopoulos $\mathrm{G}$, et al. Waldenström macroglobulinemia: clinical course and prognostic factors in 60 patients. Experience from a single hematology unit. Ann Hematol. 2001;80:722-7.

29. Lee HS, Kim K, Yoon DH, Kim JS, Bang S-M, Lee J-O, Eom HS, Lee H, Kim I, Lee WS, et al. Clinical factors associated with response or survival after chemotherapy in patients with Waldenström macroglobulinemia in Korea. Biomed Res Int. 2014;2014.

30. Treon SP. How I treat Waldenstrome Macroglobulinemia. Blood. 2015;126:721-33.

31. Byrd JC, White CA, Link B, Lucas MS, Velasquez WS, Rosenberg J, GrilloLópez AJ. Rituximab therapy in Waldenstrom's macroglobulinemia: preliminary evidence of clinical activity. Ann Oncol. 1999;10:1525-7.

32. Dimopoulos MA, Anagnostopoulos A, Kyrtsonis MC, Castritis E, Bitsaktsis A, Pangalis GA. Treatment of relapsed or refractory Waldenström's macroglobulinemia with bortezomib. Haematologica. 2005;90:1655-8.

33. Gertz MA, Rue M, Blood E, Kaminer LS, Vesole DH, Greipp PR. Multicenter phase 2 trial of rituximab for Waldenström Macroglobulinemia (WM): an eastern cooperative oncology group study (E3A98). Leukemia Lymphoma. 2004;45:2047-55.

34. Treon SP, Hanzis C, Tripsas C, loakimidis L, Patterson CJ, Manning R, Sheehy P. Bendamustine therapy in patients with relapsed or refractory Waldenström's Macroglobulinemia. Clin Lymphoma Myeloma Leukemia. 2011;11:133-5.

35. Oza A, Rajkumar SV. Waldenstrom macroglobulinemia: prognosis and management. 2015. https://doi.org/10.1038/bcj.2015.28.

\section{Publisher's Note}

Springer Nature remains neutral with regard to jurisdictional claims in published maps and institutional affiliations. 